\author{
D.S. BRITT ${ }^{1}$, M. MEDVINSKY ${ }^{2}$, E. TURKEL ${ }^{2}$, C.В. ЦЫHКOB ${ }^{1,3}$ \\ ${ }^{\prime}$ North Carolina State University, Raleigh, NC, USA, \\ dsbritt@ncsu.edu,stsynkov@ncsu.edu \\ ${ }^{2}$ Tel Aviv University, Israel, \\ medvinsk@post.tau.ac.il,turkel@post.tau.ac.il \\ ${ }^{3}$ Московский Физико-Технический Институт, Долгопрудный 141700, Россия
}

\title{
HIGH ORDER NUMERICAL SIMULATION OF THE TRANSMISSION AND SCATTERING OF WAVES USING THE METHOD OF DIFFERENCE POTENTIALS
}

Метод разностных потенциалов, предложенный Рябеньким, применён совместно с компактными разностными схемами высокого порядка точности для решения задач распространения волн в областях с переменными и, возможно, разрывными характеристиками среды.

The method of difference potentials by Ryaben'kii is combined with compact high order accurate finite difference schemes for solving the problems of propagation of waves across the regions with variable and/or discontinuous material characteristics.

We present a high order accurate methodology for the numerical simulation of time-harmonic waves governed by the variable coefficient Helmholtz equation. Our approach combines the method of difference potentials developed by Ryaben'kii [1] with compact finite difference schemes that provide an inexpensive venue toward high order accuracy [2,3]. The method of difference potentials can be interpreted as a generalized discrete version of the method of Calderon's operators in the theory of partial differential equations.

The method of difference potentials [1] offers several key advantages, such as the capability of handling boundaries/interfaces that are not aligned with the discretization grid, variable coefficients, and nonstandard boundary conditions. In doing so, the complexity of the algorithm remains comparable to that of a conventional finite difference scheme on a regular structured grid. In addition to that, compact schemes [2,3] enable high order accuracy on narrow stencils and hence require only as many boundary conditions as needed for the underlying differential equation itself.

We have applied the proposed methodology to solving several variable coefficient interior Helmholtz problems with fourth and sixth order accuracy [4]. We have also analyzed the exterior scattering of time-harmonic waves about smooth shapes, as well as a number of transmission/scattering problems [5], in which not only do the waves scatter off a given shape but also propagate through the interface and travel across the heterogeneous medium inside. In all 
the cases, our methodology guarantees high order accuracy for variable coefficients, regular grids, and non-conforming boundaries and interfaces $[4,5]$.

In addition to that, we have solved several problems with non-standard boundary conditions, such as variable coefficient Robin and mixed Dirichlet/Neumann boundary conditions [6]. A significant advantage of our approach is that it introduces a universal framework for treating the boundary conditions of any type, and that altering the boundary condition requires only minor changes to the overall algorithm.

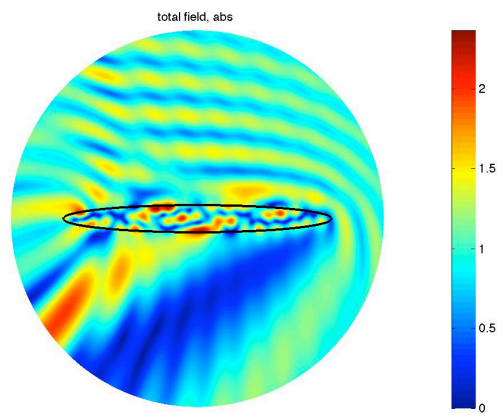

Figure 1. Scattering of a plane wave about an ellipse with aspect ratio 10. Absolute value of the total field is shown. The exterior wavenumber is 10 and the interior wavenumber is 20 .

Work supported by the US National Science Foundation, grant DMS-0810963, US Army Research Office, grant W911NF-11-1-0384, and US-Israel Binational Science Foundation, grant 2008094.

\section{Bibliography:}

[1] В.С. Рябенький, Метод разностных потенциалов и его приложения, 2-е издание, Москва, ФИЗМАТЛИТ, 2002, 496 с.

[2] S. Britt, S. Tsynkov, and E. Turkel, A Compact Fourth Order Scheme for the Helmholtz Equation in Polar Coordinates, Journal of Scientific Computing, Vol. 45, Nos. 1-3 (2010) pp. 2647.

[3] S. Britt, S. Tsynkov, and E. Turkel, Numerical Simulation of Time-Harmonic Waves in Inhomogeneous Media using Compact High Order Schemes, Communications in Computational Physics, Vol. 9, No. 3 (2011) pp. 520-541.

[4] M. Medvinsky, S. Tsynkov, and E. Turkel. The Method of Difference Potentials for the Helmholtz Equation Using Compact High Order Schemes, Journal of Scientific Computing, Vol. 53 (2012) pp. 150-193.

[5] M. Medvinsky, S. Tsynkov, and E. Turkel. High Order Numerical Simulation of the Transmission and Scattering of Waves Using the Method of Difference Potentials, Journal of Computational Physics, Vol. 243 (2013) pp. 305-322.

[6] S. Britt, S. Tsynkov, and E. Turkel. A High Order Numerical Method for the Helmholtz Equation with Non-Standard Boundary Conditions, to appear in SIAM Journal on Scientific Computing. 